\title{
Anwendung von Qualitätskennzahlen in den Niederösterreichischen Landeskliniken
}

F. Fuchs

Qualitätsmanagement

Schlüsselwörter

Ergebnisqualität

Qualitätsindikatoren

Fehlerkultur

Keywords

outcome quality

quality indicators

error culture
Institut

NÖ Landeskliniken-Holding,

St. Pölten, Österreich

\section{Bibliografie}

DOI 10.1055/s-0029-1242666 Dtsch Med Wochenschr 2009; 134: S310 - (c) Georg Thieme Verlag KG Stuttgart · New York . ISSN 0012-0472

\section{Korrespondenz}

\section{r. Fabiola Fuchs}

Leiterin der Abteilung Strat. Qualitätsentwicklung in der NÖ Landeskliniken-Holding

Stattersdorfer Hauptstraße 6C 3100 St. Pölten

Österreich

Tel. +43 (0)2742/313-813

eMail fabiola.fuchs@

holding.Iknoe.at

\section{Ausgangslage}

Die Qualitätssicherung in den österreichischen Krankenhäusern liegt im Wesentlichen in der Hand des jeweiligen Eigentümers. Die Landesfonds (in Niederösterreich der NÖGUS) sind gesetzlich zur Qualitätssicherung verpflichtet. Der NÖGUS legte den Fokus in den ersten Jahren ab Bestehen großteils auf Strukturqualität. Ab 2005 wurden durch die Erstellung von Qualitätsberichten zu bestimmten Operationen bzw. Diagnosen erste Schritte in Richtung Ergebnisqualität unternommen. Bislang gibt es jedoch noch immer kein gesamtösterreichisches Konzept zur Thematik Ergebnisqualitätsmessung, indem die einzelnen Spitäler verglichen werden können. Dadurch entschloss man sich in Niederösterreich (NÖ) 2008 zur strukturierten Ergebnismessung und somit zur selbstständigen Entwicklung von Qualitätskennzahlen und entwickelte ein Set von 38 Indikatoren aus den wichtigsten Fachbereichen. Im Zuge der Umsetzung einer neuen Qualitätsstrategie wurden dann auch Gremien wie Regionale Medizinische Beiräte und Fachbeiräte eingerichtet, in denen die Kennzahlenergebnisse besprochen werden. Ein Jahr später konnten dann Helios und das Schweizer Bundesamt für Gesundheit als Benchmarking Partner gewonnen werden.

Durch die Zusammenarbeit mit den Helios Kliniken ist das Indikatorenset inzwischen auf 98 angewachsen und misst Mortalität, Komplikationen, Intensivhäufigkeit, präoperative Verweildauer, Fehlbelegung, Anteil Tagesklinik oder reine Mengen aus verschiedenen Fachbereichen. Die endgültige Abstimmung der Inhalte fand Mitte Juli statt und zurzeit erfolgt der endgültige Check aus fachlicher sowie EDV-technischer Sicht. Ab 2010 werden bestimmte Indikatoren auch in die „Balanced Scorecard“ der einzelnen Kliniken übernommen.

Um die medizinökonomische Perspektive nicht außer Acht zu lassen, kamen zu den Qualitätsindikatoren die Steuerungsindikatoren Verweildauer, Tagesklinikpotenzial sowie Intensivhäufigkeit hinzu, die die 15 häufigsten Leistungen auf Abteilungsebene abbilden.

\section{Vorgehen bei Auffälligkeiten}

Die quartalsweise ausgewerteten Daten werden in den Gremien mit den ärztlichen Leitern bzw. den Abteilungsvorständen besprochen und in Bezug auf die Steuerungsindikatoren werden Zielvereinbarungen getroffen. Die Qualitätsindikatoren-Ergebnisse werden zusätzlich in den be- troffenen Fachbeiräten besprochen und Auffälligkeiten in einem Peer Review mit einheitlichen Prüfbogen analysiert.

\section{Herausforderungen}

Die große Herausforderung in der Abstimmung der Inhalte mit Helios war die Überwindung der Unterschiede zwischen dem deutschen DRG und dem österreichischen LKF(Leistungsorientierte Krankenhausfinanzierung)-System. Die operativen Leistungen konnten großteils unproblematisch abgeglichen werden. Kompliziert wurde die Abstimmung der Diagnosen durch die einerseits grundsätzlich unterschiedliche Definition der Hauptdiagnose und andererseits der nicht zufriedenstellenden Datenqualität in Bezug auf Diagnosen in Österreich. Dieses Problem sollte zu Beginn die in NÖ verwendete „ziehende MEL (Medizinische Einzelleistung)“ lösen, konnte schlussendlich jedoch nur durch komplexe Ausschlusskriterien behoben werden. Der Druck zu Punkteoptimierungen ist noch immer gegeben und schränkt die qualitative Betrachtung in Bezug auf die Verweildauer oder Intensivhäufigkeit stark ein. Die transparente Ergebnisdarstellung stellt für die Abteilungsvorstände noch immer eine Bedrohung dar und die notwendige Fehlerkultur hat in Österreich noch nicht Einzug erhalten.

In den vergangenen Monaten wurde durch massiven medialen Druck immer wieder über die Veröffentlichung der Ergebnisse diskutiert. Dieser Gang in die Öffentlichkeit wird in Zusammenarbeit mit der Patientenanwaltschaft und den Fachexperten vorbereitet.

Autorenerklärung: Die Autorin erklärt, dass keine relevanten finanziellen Verbindungen in Bezug auf dieses Manuskript bestehen. 